\title{
COMPUTATIONAL APPROACH TO THE EXPERIMENTAL DETERMINATION OF DIFFUSION COEFFICIENTS FOR OXYGEN AND NITROGEN IN HYDRAULIC FLUIDS USING THE PRESSURE-DECAY METHOD
}

\author{
Andris Rambaks*, Filipp Kratschun, Carsten Flake, Maren Messirek, Katharina Schmitz, \\ Hubertus Murrenhoff \\ Institute for Fluid Power Drives and Systems, RWTH Aachen University, Campus-Boulevard 30, 52074 Aachen \\ Chair of Technical Thermodynamics, RWTH Aachen University, Schinkelstraße 8, 52062 Aachen \\ * Corresponding author: Tel.: +49 241 8047723; E-mail address: andris.rambaks@ifas.rwth-aachen.de
}

\begin{abstract}
In the presented paper, the applicability of pressure-decay methods to determine the diffusivities of gases in hydraulic fluids is analysed. First, the method is described in detail and compared to other measurement methods. Secondly, the thermodynamics and the mass transfer process of the system are studied. This results in four different thermodynamic models of the gaseous phase in combination with two diffusion models. Thirdly, the influence of the models on the pressure-decay method is evaluated computationally by examining the diffusion process of air in water as all system parameters are available from literature. It is shown that ordinary pressure-decay methods are not applicable to gas mixtures like air and therefore a new method for calculating the diffusivities is suggested.
\end{abstract}

Keywords: Diffusivity, Pressure-decay method, Binary and ternary diffusion, Virial equation of state

\section{INTRODUCTION}

At all times in hydraulic systems a certain amount of air is present. It takes the form of entrained gaseous bubbles as well as dissolved air. Entrained air increases the risk of cavitation damage and reduces the effective bulk modulus of the fluid [1]. Dissolved air normally has no effect on the properties of the fluid but under certain circumstances it can outgas and increase the amount of entrained air in the system $[1,2]$. Therefore, it is of interest to determine the amount of dissolved air as well as the speed of the diffusion process.

The amount of gas solved in an ideal dilute solution is dependent on Henry's law constant and the partial pressure of the gas [3] given by equation (1):

$$
x_{i}^{\prime \prime} \cdot p^{\prime \prime}=H_{i k} \cdot x_{i}^{\prime}
$$

In hydraulics, the Bunsen absorption coefficient is often used instead and is given by equation (2):

$$
\alpha_{i}=\frac{\left(V_{i}^{\prime}\right)_{S}}{\left(V_{k}^{\prime}\right)_{S}} \cdot \frac{(p)_{S}}{p_{i}^{\prime \prime}}
$$

The speed of the diffusion process depends on the diffusivity of the considered system. For binary system the diffusion process is governed by Fick's $2^{\text {nd }}$ law of diffusion given by equation (3):

$$
\frac{\partial C_{i}^{\prime}}{\partial t}=D_{i k} \cdot \frac{\partial^{2} C_{i}^{\prime}}{\partial x^{2}}
$$

Diffusion processes involving more than two solutes can be modelled with the generalized Fick's law or the Maxwell-Stefan equations. Due to their ease of applicability, the Maxwell-Stefan equations are chosen in this research (4):

$$
\sum_{\substack{j=1 \\ j \neq i}}^{N} \frac{x_{i}^{\prime} \cdot x_{j}^{\prime}}{\bigoplus_{i j}}\left(\frac{j_{i}^{\prime}}{C_{i}^{\prime}}-\frac{j_{j}^{\prime}}{C_{j}^{\prime}}\right)=-\frac{\partial x_{i}^{\prime}}{\partial x} \quad \forall i
$$

Currently there is only limited data available for diffusion coefficients of gases in hydraulic fluids. In this paper the authors address the problems involved in estimating the diffusivities for high pressure systems for ideal and non-ideal gases and gas mixtures. The emphasis is placed on the diffusion of nitrogen and oxygen in hydraulic fluids at pressures up to 300 bar. Thus, 
the theory of diffusion for binary as well as ternary systems is analysed and their applicability to high pressure conditions is discussed. The three questions to be answered by this paper are:

1. Which method is to be preferred to measure the diffusion coefficients of gases in hydraulic fluids?

2. Is it necessary to regard air as a real gas or can the assumption of an ideal gas be made?

3. Can changes in the molar fractions of the gaseous phase be neglected?

4. How can the diffusivity of oxygen be determined for hydrocarbon-based hydraulic fluids?

\section{MEASUREMENT METHODS AND APPARATUS}

Several measurement methods for determining the diffusion coefficients of gases in liquids exist, originating from the research of crude oil. The measurement methods are typically categorized into two groups: direct and indirect methods.

Direct methods require measuring concentration profiles of diffusing components in order to directly determine diffusion coefficients. These methods involve either highly error-prone sampling, especially under high pressures [4], or non-invasive analysis techniques, which are usually expensive and demanding, like nuclear magnetic resonance [5], computer-assisted tomography $[6,7]$ or planar laser-induced fluorescence $[8,9]$.

Indirect methods require the observation of phenomena which are influenced by diffusion in order to indirectly determine the diffusivities. Indirect methods include decreasing bubble size methods [10] and constant bubble size methods $[11,12]$. As the names indicate the volume of a gas bubble inside the liquid phase is observed. The size can be decreasing due to absorption and diffusion of the gas into the liquid phase or the size can be constant if an appropriate gas volume flow into the bubble is provided. Also, indirect methods exist, for which the gas and liquid phase are reversed and the volume [13] or shape [14] of a liquid drop in a gas atmosphere is analysed. Bubble and drop size methods are relatively fast (experimental time within hours) and only need small sample sizes. The drawbacks are the elaborate techniques to isolate and fix the bubbles and drops without deforming them. Similar to drop and bubble size methods are microfluidic approaches in which the change of the interface due to swelling is observed [15].

Most widely used are pressure decay methods [16-22], which is the method of choice in this paper. The advantages of this method are its simplicity and applicability to wide ranges of temperatures and especially pressures. Furthermore, with the same method it is also possible to accurately determine the Bunsen absorption coefficient as shown by Rambaks et al. [23]. A picture of the apparatus is shown in Figure 1, the working principles are presented Figure 2.

A cylindrical measurement chamber is partially filled with a specified volume of hydraulic fluid and connected to a compressed air bottle. With time nitrogen and oxygen will dissolve into the hydraulic fluid until it is completely saturated. At this point in time thermodynamic equilibrium (State 0 ) is reached. Subsequently, the piston of the apparatus is used to compress the measurement chamber further to State 1 . This is a non-equilibrium state, at which it is assumed that the diffusion process begins and persists until a new thermodynamic equilibrium has been reached in State 2. [23]

During the diffusion process, the amount of gas in the gaseous phase decreases due to the increased solubility of the liquid at higher pressures. Therefore, a drop in pressure over time is measured and used to calculate the diffusion coefficient.

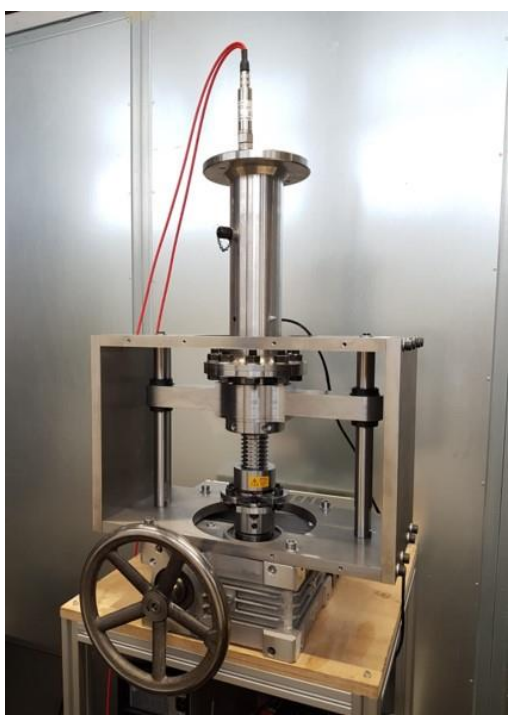

Figure 1: Pressure-decay measurement apparatus 


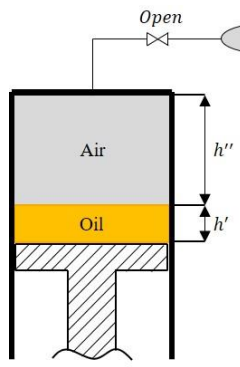

Gasification

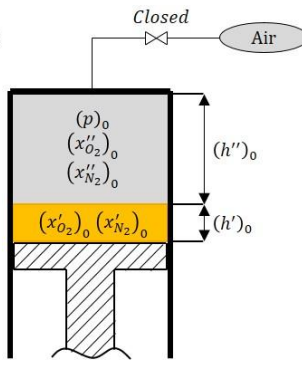

State: 0

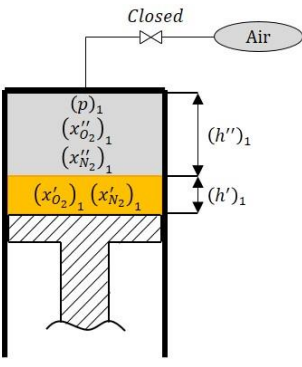

State: 1

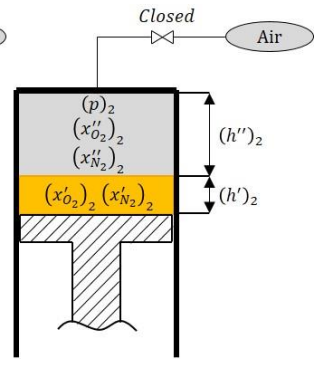

State: 2

Figure 2: Working principle of the measurement system [23]

\section{THERMODYNAMICS AND MASS TRANSFER}

The diffusion process is governed either by Fick's $2^{\text {nd }}$ law of diffusion for binary systems (3) or by the Maxwell-Stefan equations (4) if more than two species are involved in the diffusion process.

For binary systems the diffusivity of gases can be determined easily from the measured pressure-decay curves [16-22], however for some systems this approach is not applicable due to safety reasons. The prime example of this is the diffusion of oxygen in hydrocarbon-based hydraulic fluids, which poses ignition risks.

The diffusivity of oxygen in hydraulic fluids could be determined by examining the diffusion of air, but the pressure-decay curve does not provide information about the individual diffusivities of oxygen and nitrogen. To formulate a new method for estimating the diffusivities of individual gas species, different diffusion models are analysed in the following chapters. Prior to that the solubility of gases in liquids is discussed.

\subsection{Solubility of gases in liquids}

According to Lüdecke et al. [3], thermodynamic equilibrium of a two-phase closed system persists, if it is in mechanical (5), thermal (6) and its components are in chemical equilibrium (7).

$$
\begin{gathered}
p^{\prime}=p^{\prime \prime} \\
T^{\prime}=T^{\prime \prime} \\
\mu_{i}^{\prime}=\mu_{i}^{\prime \prime} \quad \forall i
\end{gathered}
$$

In an equilibrium state of the two-phase system, a certain amount of dissolved gas is present in the liquid phase. To calculate the initial values of the concentration of dissolved gas in the liquid phase, the Bunsen absorption coefficient (2) for each species of gas has to be determined.

In Rambaks et al. [23] a method to calculate the Bunsen absorption coefficient for gases at high pressures with the use of measurement data from the equilibrium states was presented. It was found that the Bunsen absorption coefficient (2) can be calculated by evaluating the change in mass of gas in the gaseous phase given by equation (8):

$$
\alpha_{i}=\left(\Delta m_{i}^{\prime \prime}\right)_{0 \rightarrow n} \cdot \frac{\left(R_{i}\right)_{S} \cdot(T)_{S}}{\left(p_{i}^{\prime \prime}\right)_{n} \cdot\left(V_{k}^{\prime}\right)_{S}}
$$

The equation for the change in mass of gas in the gaseous phase is dependent on the pressure and temperature range and if the gas is a single species gas or a gas mixture. The reader is referred to Rambaks et al. [23] for a detailed analysis.

\subsection{Binary diffusion model of ideal single species gases}

The principle of binary diffusion within the context of the apparatus is depicted in Figure 3. The process is governed by Fick's $2^{\text {nd }}$ law of diffusion(3). The initial values for the concentration as well as appropriate boundary conditions must be specified to solve the partial differential equation given by (3). 


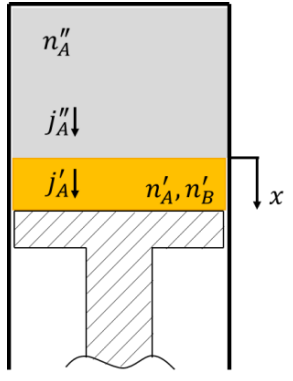

Figure 3: Binary diffusion in measurement apparatus

According to Kratschun et al. [24] and Freudigmann et al. [25] in the timescale of short deviations from a thermodynamic equilibrium of the system the diffusion process in negligible. Consequently, this allows the assumption that during the compression from State 0 to State 1 diffusion is negligible, and that the diffusion process begins only when State 1 has been reached. Therefore, the initial values for species concentration within the liquid phase can be determined based on equation (9):

$$
C_{i}^{\prime}(x, t=0)=\alpha_{i} \cdot \frac{\left(p^{\prime \prime}\right)_{0}}{\bar{R} \cdot(T)_{s}} \quad \forall x \in(0, L)
$$

Because of the diffusion process, the amount of substance and, therefore, the pressure in the measurement chamber decreases. The relationship between pressure, volume, temperature and amount of substance can be described with the equation of state for an ideal single species gas given by equation (10):

$$
p_{i}^{\prime \prime} \cdot V^{\prime \prime}=n_{i}^{\prime \prime} \cdot \bar{R} \cdot T^{\prime \prime}
$$

The temporal change in the amount of substance in the gaseous phase can be expressed by taking the derivative of equation (10) with respect to time, resulting in equation (11):

$$
\frac{d n_{i}^{\prime \prime}}{d t}=\frac{V^{\prime \prime}}{\bar{R} \cdot T^{\prime \prime}} \cdot \frac{d p_{i}^{\prime \prime}}{d t}
$$

By constructing a control volume around the gaseous phase, the molar flux within the gaseous phase can be determined with equation (12):

$$
j_{i}^{\prime \prime}=-\frac{4}{\pi \cdot d^{2}} \cdot \frac{d n_{i}^{\prime \prime}}{d t}
$$

With the use of a surface mass balance at the interface between the liquid and gaseous phase, the molar flux within the liquid phase is equal to the molar flux within the gaseous phase and can be expressed with expression (13):

$$
j_{i}^{\prime}=j_{i}^{\prime \prime}
$$

From equations (12) and (13) it can be derived, that the molar flux of dissolved gas within the liquid phase is given by equation (14):

$$
j_{i}^{\prime}=-\frac{4}{\pi \cdot d^{2}} \cdot \frac{d n_{i}^{\prime \prime}}{d t}
$$

According to Fick's $1^{\text {st }}$ law of diffusion, the molar flux is directly proportional to the diffusivity and the concentration gradient as given by equation (15):

$$
j_{i}^{\prime}=-D_{i k} \cdot \frac{\partial C_{i}^{\prime}}{\partial x}
$$

Equation(15) allows to define Neumann boundary conditions at both ends of the liquid phase. At the gas-liquid interface, no accumulation of substance is possible. Therefore, the concentration gradient at the interface can be expressed with equation (16):

$$
\left.\frac{\partial C_{i}^{\prime}}{\partial x}\right|_{x=0}=\frac{4}{\pi \cdot d^{2} \cdot D_{i k}} \cdot \frac{d n_{i}^{\prime \prime}}{d t}
$$

Besides the Neumann boundary condition (16), a Dirichlet boundary condition given by equation (17) is specified:

$$
C_{i}^{\prime}(x=0, t)=\alpha_{i} \cdot \frac{p^{\prime \prime}(t)}{\bar{R} \cdot(T)_{s}}
$$

At the liquid-piston interface, there is no molar flux present and consequently the Neumann boundary condition is given by equation (18):

$$
\left.\frac{\partial C_{i}^{\prime}}{\partial x}\right|_{x=L}=0
$$

With equations (9), (16), (17) and (18) the transient diffusion equation (3) is fully defined and can be solved even analytically for certain cases [19].

\subsection{Binary diffusion model of real single species gases}

According to Lüdecke et al. [3], the ideal gas law is applicable only in small pressure and temperature ranges. To take these limitations into account, a real gas model must be used. For single species gases it is convenient to use the compressibility factor, which is readily available from literature (see Vasserman et al. [28]). 
The compressibility factor describes, how much a real gas deviates from the behaviour of an ideal gas. It is given by equation (19):

$$
z_{i}=\frac{p_{i}^{\prime \prime} \cdot V^{\prime \prime}}{n_{i}^{\prime \prime} \cdot \bar{R} \cdot T}
$$

By using equation (19) and the chain rule, an expression for the temporal change in the amount of substance in the gaseous phase is found (20):

$\frac{d n_{i}^{\prime \prime}}{d t}=\frac{V^{\prime \prime}}{\bar{R} \cdot T^{\prime \prime} \cdot z_{i}^{2}}\left(z_{i}-p^{\prime \prime} \cdot \frac{d z_{i}}{d p_{i}^{\prime \prime}}\right) \frac{d p_{i}^{\prime \prime}}{d t}$

Because the measurements are taken at high pressures, this approach is necessary due to large deviations from the ideal gas law as can be seen in Figure 4.

\subsection{Ternary diffusion model of ideal gas mixtures}

In the previous sections the theory of binary diffusion was discussed. This theory is applicable if the diffusion process involves the solvent and only one solute from the gaseous phase. It cannot be applied if two or more solutes are involved in the diffusion process at the same time [27].

Air is a multicomponent gas mixture consisting primarily of nitrogen and oxygen. Additionally, other gases like argon, carbon dioxide, neon, helium, water vapour and methane can also be present [3]. As an approximation, air can be treated as a two-component gas mixture consisting of nitrogen and oxygen. This approach is adopted as it significantly simplifies the model.

Because the diffusion process involves three components (ternary diffusion), the MaxwellStefan equations (4) must be used. Using relations for the concentration and molar fractions equation(4) is rearranged to give equation (21):

$$
\sum_{\substack{j=1 \\ j \neq i}}^{N} \frac{x_{j}^{\prime} \cdot j_{i}^{\prime}-x_{i}^{\prime} \cdot j_{j}^{\prime}}{\bigoplus_{i j}}=-C^{\prime} \cdot \frac{\partial x_{i}^{\prime}}{\partial x} \quad \forall i
$$

According to Whitaker [27], for ideal dilute solutions the assumptions given by equation (22) can be made for a solvent $k$ :

$$
\begin{array}{ccc}
x_{N_{2}}^{\prime} \rightarrow 0 & \Rightarrow & C_{N_{2}}^{\prime} \rightarrow 0 \\
x_{O_{2}}^{\prime} \rightarrow 0 & \Rightarrow & C_{O_{2}}^{\prime} \rightarrow 0 \\
x_{k}^{\prime} \rightarrow 1 & \Rightarrow & C^{\prime}=C_{k}^{\prime}=\text { const } .
\end{array}
$$

These assumptions allow to simplify the Maxwell-Stefan equations to obtain expressions (23) and (24):

$$
\begin{gathered}
\frac{\partial C_{N_{2}}^{\prime}}{\partial x}=-\frac{j_{N_{2}}^{\prime}}{\bigoplus_{N_{2} k}} \\
\frac{\partial C_{O_{2}}^{\prime}}{\partial x}=-\frac{j_{O_{2}}^{\prime}}{\bigoplus_{O_{2} k}}
\end{gathered}
$$

According to Bird et al. [26], the temporal changes of concentration in the liquid phase are governed by equations (25) and (26):

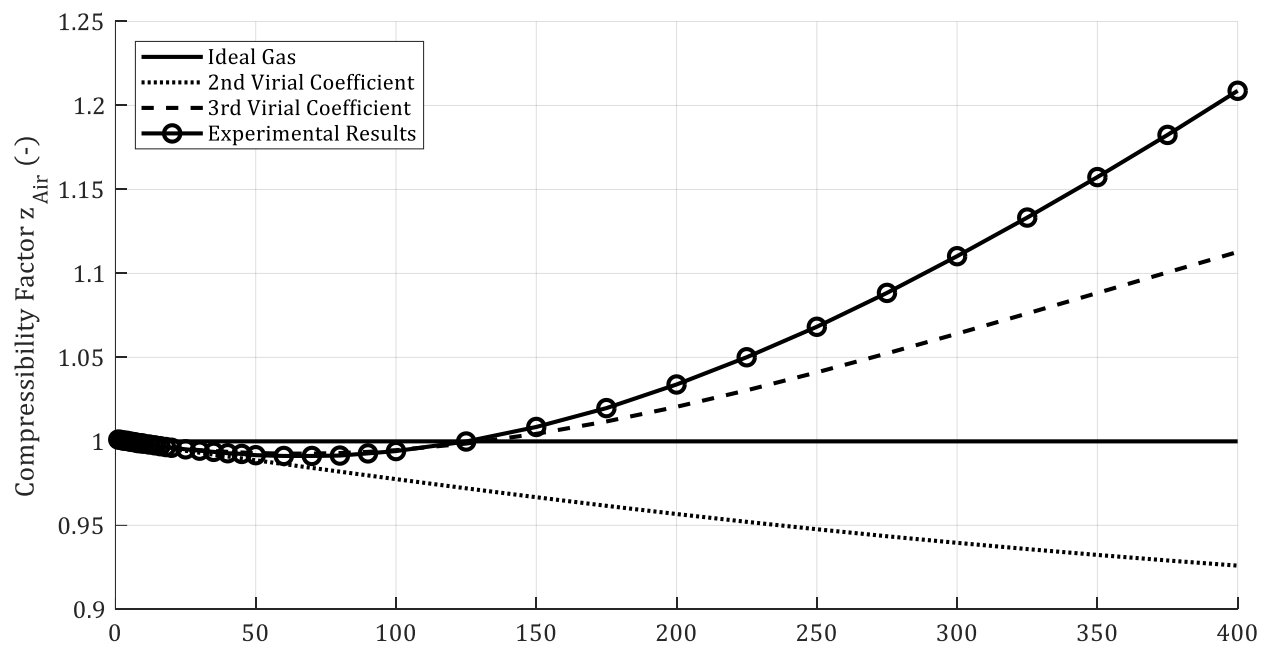

Figure 4: Compressibility factors for air [28] 


$$
\begin{aligned}
& \frac{\partial^{2} C_{N_{2}}^{\prime}}{\partial x^{2}}=-\frac{1}{\bigoplus_{N_{2} k}} \cdot \frac{\partial j_{N_{2}}^{\prime}}{\partial x} \\
& \frac{\partial^{2} C_{O_{2}}^{\prime}}{\partial x^{2}}=-\frac{1}{\bigoplus_{O_{2} k}} \cdot \frac{\partial j_{O_{2}}^{\prime}}{\partial x}
\end{aligned}
$$

By differentiating (23) and (24) with respect to $x$ and by using equations (25) and (26) expressions for the transient diffusion of nitrogen and oxygen are obtained:

$$
\begin{aligned}
& \frac{\partial C_{N_{2}}^{\prime}}{\partial t}=D_{N_{2} k} \cdot \frac{\partial^{2} C_{N_{2}}^{\prime}}{\partial x^{2}} \\
& \frac{\partial C_{O_{2}}^{\prime}}{\partial t}=D_{O_{2} k} \cdot \frac{\partial^{2} C_{O_{2}}^{\prime}}{\partial x^{2}}
\end{aligned}
$$

As can be observed from expressions (27) and (28), the complex Maxwell-Stefan equations reduce to two equations of binary diffusion. The reason for this is the assumption of an ideal dilute solution in which interactions between the solutes do not occur [26]. These assumptions are only possible because the concentration of solvent is almost unity compared to the concentration of the gases to be dissolved. In other words, due to the low solubility of the air's components the Maxwell-Stefan equations converge to Fick's $2^{\text {nd }}$ law but for oxygen and nitrogen separately.

If the diffusivity is to be measured at moderate pressures and temperatures, the ideal gas mixture model can be used. Then, using Dalton's law the temporal changes in the amount of substance of nitrogen and oxygen can be expressed with equations (29) and (30):

$$
\begin{aligned}
& \frac{d n_{N_{2}}^{\prime \prime}}{d t}=\frac{V^{\prime \prime}}{\bar{R} \cdot T^{\prime \prime}} \cdot \frac{d p_{N_{2}}^{\prime \prime}}{d t} \\
& \frac{d n_{O_{2}}^{\prime \prime}}{d t}=\frac{V^{\prime \prime}}{\bar{R} \cdot T^{\prime \prime}} \cdot \frac{d p_{O_{2}}^{\prime \prime}}{d t}
\end{aligned}
$$

\subsection{Ternary diffusion model of real gas mixtures}

When considering the solubility of a real gas mixture, the fugacity coefficient has to be taken into account due to the non-ideal behaviour of the gaseous phase [3]. Hence, equation (1) is rewritten to equation (31):

$$
x_{i}^{\prime \prime} \cdot \varphi_{i} \cdot p^{\prime \prime}=H_{i k} \cdot x_{i}^{\prime} \quad \forall i
$$

According to Lüdecke et al. [3], multiple equations of state can be used to calculate the fugacity coefficient of each gas species. In this paper, the virial equation of state with 3 coefficients is chosen and is given by expression (32):

$$
p^{\prime \prime} \cdot v_{m}^{\prime \prime}=\bar{R} \cdot T^{\prime \prime} \cdot\left(1+\frac{B}{v_{m}^{\prime \prime}}+\frac{C}{v_{m}^{\prime \prime 2}}\right)
$$

The equation for the fugacity coefficient is given by equation (33):

$$
\begin{aligned}
\ln \left(\varphi_{i}\right)= & \frac{2}{v_{m}^{\prime \prime}} \sum_{j=1}^{N} x_{j}^{\prime \prime} B_{i j}-\ln (z) \\
& +\frac{3}{2 v_{m}^{\prime \prime 2}} \sum_{j=1}^{N} \sum_{k=1}^{N} x_{j}^{\prime \prime} x_{k}^{\prime \prime} C_{i j k}
\end{aligned}
$$

Using equation (31) the necessary Dirichlet boundary condition for each species of gas can be calculated with expression (34):

$$
x_{i}^{\prime}(x=0, t)=\frac{x_{i}^{\prime \prime} \cdot \varphi_{i} \cdot p^{\prime \prime}}{H_{i, k}}
$$

The pressure-decay method allows to calculate the diffusivity of pure gases, however the diffusivity of oxygen cannot be determined in this way due to ignition risks. Therefore, the authors propose a new approach:

1. Calculate the diffusivity of pure nitrogen;

2. Measure the pressure-decay for air;

3. Determine the diffusivity of oxygen from the pressure-decay curve for air with computational simulations using the ternary diffusion model of real gas mixtures.

\section{COMPUTATIONAL ANALYSIS}

To demonstrate the discrepancies between the different thermodynamic and diffusion models, simulations were conducted using a finite difference scheme. The liquid domain is discretised and an explicit Euler approach is chosen to calculate the concentration within it over time. The test case considered is the diffusion of nitrogen and oxygen in water, since the values for diffusivities and Henry coefficients are readily available. Also, the thermophysical properties of air are well documented by Vasserman et al. [28].

The single species real gas model was used to calculate the pressure-decay for nitrogen and oxygen separately. The two curves provide an 
upper and lower bound for the pressure-decay curves of the ternary diffusion models.

The parameters for the diffusivities, Henry coefficients and virial coefficients are presented in Table 1:

Table 1: Diffusivities, Henry and virial coefficients

\begin{tabular}{|c|c|}
\hline Diffusivities of system & Value $\left[\mathrm{m}^{2} / \mathrm{s}\right]$ \\
\hline Diffusivity $D_{\mathrm{N}_{2} \mathrm{H}_{2} \mathrm{O}}$ & $2.000 \times 10^{-9}$ \\
\hline Diffusivity $\mathrm{D}_{\mathrm{O}_{2} \mathrm{H}_{2} \mathrm{O}}$ & $2.420 \times 10^{-9}$ \\
\hline Henry coefficients & Value $[\mathrm{Pa}]$ \\
\hline Henry coefficient $\mathrm{H}_{\mathrm{N}_{2} \mathrm{H}_{2} \mathrm{O}}$ & $8.59 \times 10^{9}$ \\
\hline Henry coefficient $\mathrm{H}_{\mathrm{O}_{2} \mathrm{H}_{2} \mathrm{O}}$ & $4.42 \times 10^{9}$ \\
\hline Virial coefficients & Value $\left[\mathrm{m}^{3} / \mathrm{mol}\right]$ \\
\hline Coefficient $B_{N_{2} N_{2}}$ & $-4.34 \times 10^{-6}$ \\
\hline Coefficient $B_{\mathrm{N}_{2} \mathrm{O}_{2}}$ & $-9.70 \times 10^{-6}$ \\
\hline Coefficient $\mathrm{B}_{\mathrm{O}_{2} \mathrm{O}_{2}}$ & $-15.96 \times 10^{-6}$ \\
\hline Virial coefficients & Value $\left[\mathrm{m}^{6} / \mathrm{mol}^{2}\right]$ \\
\hline Coefficient $C_{N_{2} N_{2} N_{2}}$ & $1.90 \times 10^{-9}$ \\
\hline Coefficient $C_{\mathrm{N}_{2} \mathrm{~N}_{2} \mathrm{O}_{2}}$ & $1.50 \times 10^{-9}$ \\
\hline Coefficient $C_{\mathrm{N}_{2} \mathrm{O}_{2} \mathrm{O}_{2}}$ & $0.39 \times 10^{-9}$ \\
\hline Coefficient $C_{\mathrm{O}_{2} \mathrm{O}_{2} \mathrm{O}_{2}}$ & $35.00 \times 10^{-12}$ \\
\hline
\end{tabular}

The parameters of the measurement chamber are listed in Table 2:

Table 2: Dimensions of measurement chamber

\begin{tabular}{ll}
\hline Chamber parameters & Value $[\mathrm{mm}]$ \\
\hline Initial gaseous phase height $h_{0}^{\prime \prime}$ & 10.0 \\
Initial liquid phase height $h_{0}^{\prime}$ & 150.0 \\
Piston diameter $d$ & 65.0 \\
\hline
\end{tabular}

The system is considered isothermal at a temperature of $303.15 \mathrm{~K}$. The initial molar fractions in the gaseous phase are $79.38 \%$ nitrogen, $20.62 \%$ oxygen for the ternary systems. Because the solvent in this test case is water, water vapour is formed in the gaseous phase and must be considered. The values for the initial pressure (State 0), the intermediate pressure (State 1) and the saturation pressure of water [29] are given in Table 3:

Table 3: Values for pressure

\begin{tabular}{ll}
\hline Chamber pressures & Value [bar] \\
\hline Initial pressure $p_{0}^{\prime \prime}$ & 50.0 \\
Intermediate pressure $p_{1}^{\prime \prime}$ & 300.0 \\
Saturation pressure of water & $42.47 \times 10^{-3}$ \\
$p_{\mathrm{H}_{2} \mathrm{O}}^{S}(@ 303.15 \mathrm{~K})$ & \\
\hline
\end{tabular}

The molar fraction of water in the gaseous phase can be determined with Raoult's law (35):

$x_{\mathrm{H}_{2} \mathrm{O}}^{\prime \prime}\left(x_{\mathrm{H}_{2} \mathrm{O}}^{\prime} \rightarrow 1\right)=\frac{x_{\mathrm{H}_{2} \mathrm{O}}^{\prime} \cdot p_{\mathrm{H}_{2} \mathrm{O}}^{S}(303.15 \mathrm{~K})}{p_{0}^{\prime \prime}}$

The values of the molar fractions of water in the gaseous phase at $1.0 \mathrm{bar}$, in State 0 and in State 1 are given in Table 4:

Table 4: Values for molar fraction of water in air for system air-water

\begin{tabular}{ll}
\hline Molar fractions of water & Value [-] \\
\hline Molar fraction $x_{\mathrm{H}_{2} \mathrm{O}}^{\prime \prime}(@ 1$ bar $)$ & $42.47 \times 10^{-3}$ \\
Molar fraction $x_{\mathrm{H}_{2} \mathrm{O}}^{\prime \prime}($ 50 bar $)$ & $8.49 \times 10^{-4}$ \\
Molar fraction $x_{\mathrm{H}_{2} \mathrm{O}}^{\prime}(@ 300$ bar $)$ & $1.42 \times 10^{-4}$ \\
\hline
\end{tabular}

As can be seen, the water vapour content is significant only at ambient pressure. At higher pressures it is negligible compared to the content of nitrogen and oxygen. Even more interesting is that by considering this test case an upper bound for the molar fraction of water vapour in the gaseous phase is found for all other liquid-air systems.

When considering other fluids, e.g. hydraulic fluids, where the water content in the liquid phase is unknown, the limiting case of Raoult's law (35) for the water-air system is very convenient to use. Applying it to other systems is equivalent to having a relative humidity of $100 \%$ of the gaseous phase. Therefore, if in the limiting case given by (35) the molar fraction of water vapour is already negligible, it is also negligible for any other case.

With the given parameters it is possible to simulate the diffusion process and express the pressure in the measurement chamber as a function of time. A comparison between the pressure curves is given in Figure 5. As mentioned before the pressure-decay curves of pure nitrogen and pure oxygen provide an upper and lower bound for the ternary model.

As shown, the pressure curves for the ideal and real gas mixture models differ for the given parameters by a maximal value of approximately 10 bar (@190h). Once thermodynamic equilibrium is reached, the discrepancy is approximately 5 bar. Keeping in mind that the pressure-decay within the measurement chamber is recorded over time, differences in the pressure curves will lead to different values for the diffusivities. 
Another observation is depicted in Figure 6. Due to the different solubilities of nitrogen and oxygen in water, the molar fraction of nitrogen in the gaseous phase increases while that of oxygen decreases compared to their respective initial values as was already noted by Rambaks et al. [23].

A closer inspection of Figure 6 reveals that the molar fraction of nitrogen reaches a maximum value and then starts to decrease. The larger diffusion coefficient of oxygen is the most likely explanation for this observation.
The change in gaseous phase composition has a direct effect on the calculation of the diffusivities. This is most notable in equation (33) for the fugacity coefficients and in the expressions for the Dirichlet boundary conditions (34).

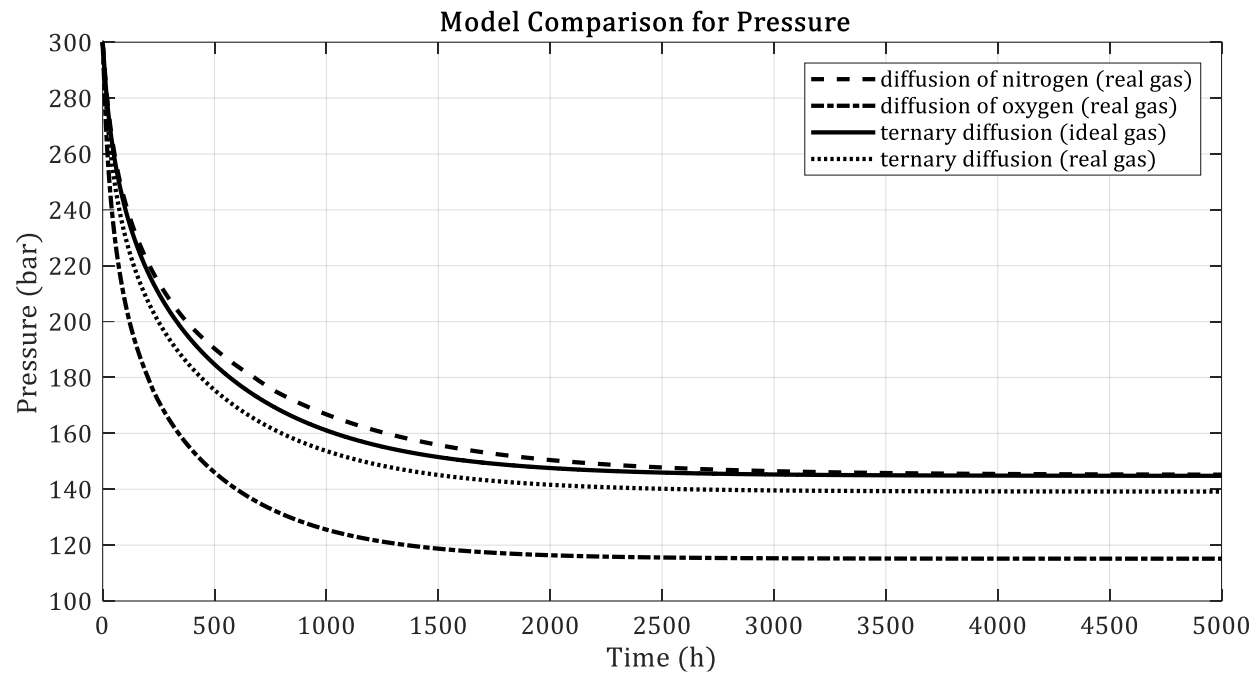

Figure 5: Pressure-decay over time for diffusion in water

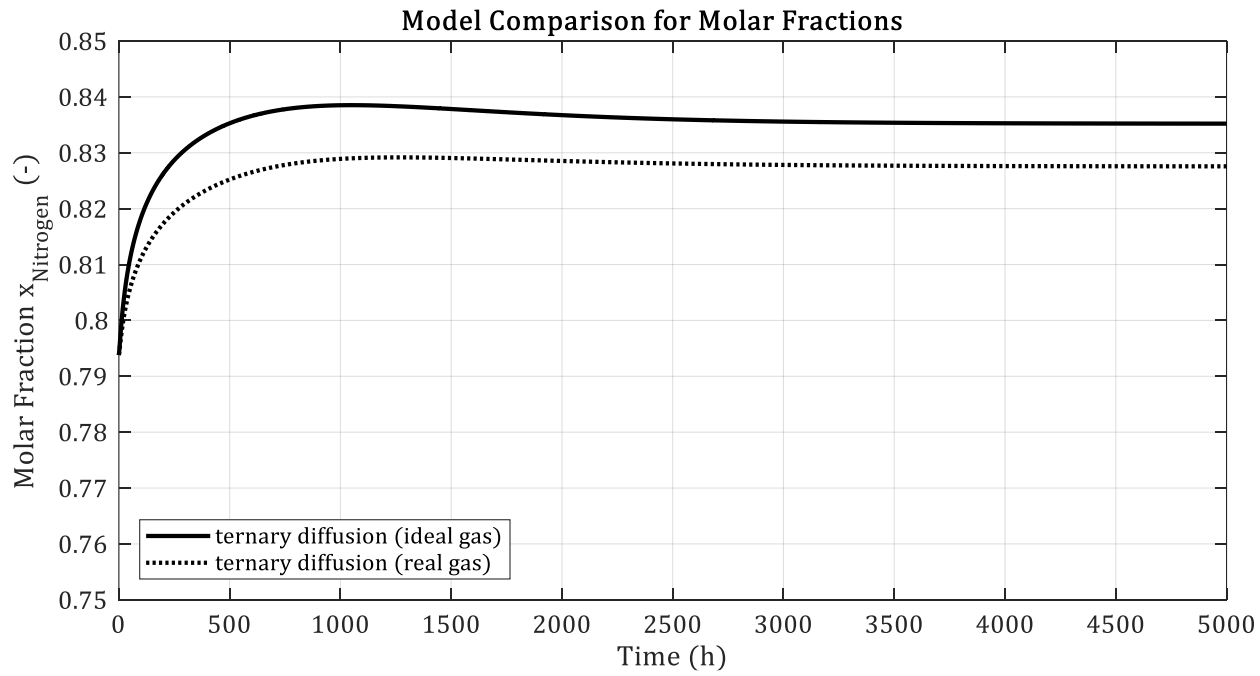

Figure 6: Molar fraction of nitrogen over time for diffusion in water 


\section{OPERATIONAL TESTING}

To test the functionality of the apparatus, a test-run with water and air at low pressure was conducted for a period of 200 hours. The measured pressure-decay curve is shown in Figure 7.

The goal of this test was to determine, if the system converges to a state of thermodynamic equilibrium. This would indicate, that the apparatus is leak-proof.

The diffusion process was accelerated with the use of a magnetic stir bar, which was placed in the measurement chamber and activated in the $12^{\text {th }}$ hour of the test. As can be observed, this leads to an immediate decrease in pressure. Following this rapid decrease, it was switched off for 10 hours and activated again within the $22^{\text {nd }}$ hour of the test. Once more, an acceleration of the diffusion process can be noted.

With time the gradient of the pressure-decay curve flattens out as depicted in Figure 7. This indicates, that the diffusion process is slowing down and converging to thermodynamic equilibrium. Based on this it can be said, that the measurement apparatus is leak-proof.

From the first 12 hours of testing the resulting preliminary pseudo-diffusivity of air in water is determined to be $D=1.1 \times 10^{-9} \mathrm{~m}^{2} / \mathrm{s}$. This value is of the same magnitude as the diffusivities of nitrogen and oxygen.

\section{CONCLUSION AND OUTLOOK}

In the presented paper different methods for determining the diffusivities of nitrogen and oxygen in liquids were discussed. Among these methods the pressure-decay method was chosen as the best suited for hydraulic fluids. A measurement apparatus and its working principles were described along with the design aspects and the operating procedure.

It was shown, that for high pressure diffusion a real gas mixture model has to be used due to the non-ideal behaviour and the changing composition of the gaseous phase. The numerical results reveal that it is necessary to capture these effects when applying the pressure decay method to gas mixtures because otherwise one cannot determine the individual diffusivities and because the non-ideal behaviour introduces severe deviations compared to the ideal gas mixture model, especially if the measurement time is limited to 10 days ( 240 hours) or less.

The described apparatus has been set up, tested and measurements of the diffusivities are subject to current research at ifas.

\section{ACKNOWLEDGMENTS}

This work was funded by the German Research Foundation (DFG) in the scope of the Project „Lösungs- und Entlösungsverhalten von Luft in Hydraulikölen" (MU 1225/41-1). The authors would like to thank the DFG for its support.

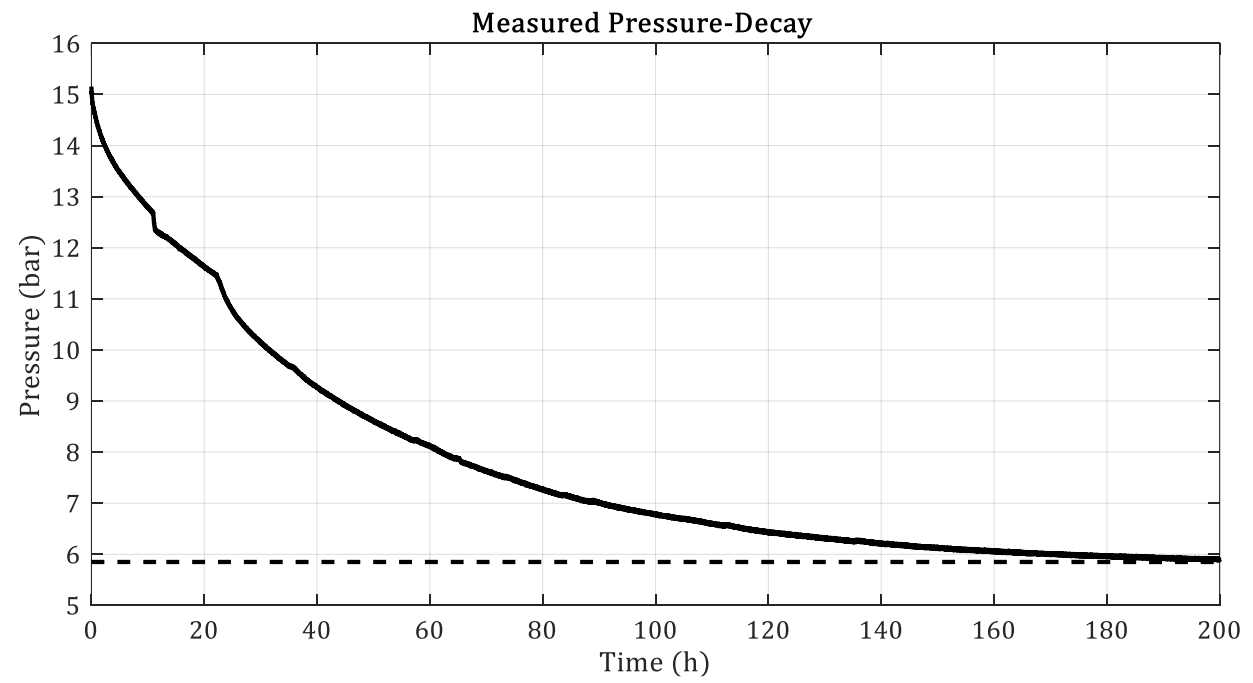

Figure 7: Measured pressure-decay over time for diffusion of nitrogen and oxygen in water 


\section{NOMENCLATURE}

$\begin{array}{ll}B & 2^{\text {nd }} \text { Virial coefficient } \\ C & \text { Concentration } / 3^{\text {rd }} \text { Virial coefficient } \\ d & \text { Piston diameter } \\ D & \text { Diffusion coefficient } \\ Đ & \text { Maxwell-Stefan-diffusion coefficient } \\ h & \text { Height } \\ H & \text { Henry coefficient } \\ i & \text { Index } \\ j & \text { Index / Molar flux } \\ k & \text { Index } \\ L & \text { Length } \\ m & \text { Mass } \\ n & \text { Index / Amount of substance } \\ N & \text { Index } \\ p & \text { Pressure } \\ R & \text { Specific gas constant } \\ \bar{R} & \text { Universal gas constant } \\ S & \text { Standard reference conditions / Saturation } \\ t & \text { Time } \\ T & \text { Temperature } \\ v m & \text { Molar Volume } \\ V & \text { Volume } \\ x & \text { Coordinate / Molar fraction } \\ z & \text { Compressibility factor } \\ \alpha & \text { Bunsen absorption coefficient } \\ \mu & \text { Chemical potential } \\ \varphi & \text { Fugacity coefficient } \\ \| & \text { Liquid phase } \\ & \text { Gaseous phase } \\ & \end{array}$

\section{REFERENCES}

[1] Schrank K (2015) Eindimensionale Hydrauliksimulation mehrphasiger Fluide. Dissertation, RWTH Aachen University

[2] Schmitz K, Murrenhoff H (2018) Grundlagen der Fluidtechnik: Hydraulik. Aachen, Germany

[3] Lüdecke C, Lüdecke D (2000) Thermodynamik: Physikalisch-chemische Grundlagen der thermischen Verfahrenstechnik. Berlin, Germany

[4] Peper S, Dohrn R (2012) Sampling from fluid mixtures under high pressure: Review case study and evaluation. The Journal of Supercritical Fluids 66: 2-15.

doi: 10.1016/j.supflu.2011.09.021

[5] Wen Y, Bryan J, Kantzas A (2005) Estimation of Diffusion coefficients in bitumen solvent mixtures as derived from low field NMR spectra. Journal of Canadian Petroleum Technology 44: 29-35. doi: 10.2118/05-04-03
[6] Wen YW, Kantzas A (2005) Monitoring bitumen-solvent interactions with low-field nuclear magnetic resonance and X-ray. Energy Fuels 4: 1319-1326. doi: 10.1021/ef049764g

[7] Li X, Song Y, Wu B, Liu Y, Jiang L (2017) Determination of swelling effect in $\mathrm{CO}_{2}$-brine systems using microfocus X-ray CT. Energy Procedia 142: 3344-3349. doi: 10.1016/j.egypro.2017.12.468

[8] Xu F, Jimenez M, Dietrich N, Hébrard G (2017) Fast determination of gas-liquid Diffusion coefficient by an innovative double approach. Chemical Engineering Science 170: 68-76. doi: 10.1016/j.ces.2017.02.043

[9] Jimenez M, Nicolas D, Cockx A, Hébrard G (2013) Experimental study of $\mathrm{O}_{2}$ Diffusion coefficient measurement at a planar gas-liquid interface by planar laser-induced fluorescence with inhibition. AIChE J, 59: 325-333. doi: 10.1002/aic.13805

[10] Kireger IM, Mulholland GW, Dickey CS (1967) Diffusion coefficients for gases in liquids from the rates of solution of small gas bubbles. The Journal of Physical Chemistry 71: 1123-1129

[11] De Blok WJ, Fortuin JMH (1981) Method for determining Diffusion coefficients of slightly soluble gases in liquids. Chemical Engineering Science 36 (10): 1687-1694. doi: 10.1016/00092509(81)80014-0

[12] Ng WY, Walkley J (2011) Diffusion of gases in liquids: the constant size bubble method. Canadian Journal of Chemistry 47: 1075-1077. doi: 10.1139/v69-170

[13] Yang C, Gu Y (2005) New experimental Method for measuring gas diffusivity in heavy oil by the dynamic pendant drop volume analysis (DPDVA). Industrial \& Engineering Chemistry Research 44: 4474-4483. doi: 10.1021/ie0501430

[14] Yang C, Gu Y (2006) A new method for measuring solvent diffusivity in heavy oil by dynamic pendant drop shape analysis (DPDSA). SPE Journal 11: 48-57. doi: 10.2118/84202-PA

[15] Fadaei H, Scarff B, Sinton D (2011) Rapid microfluidics-based measurement of $\mathrm{CO}_{2}$ diffusivity in bitumen. Energy Fuels 25: 48294835. doi: 10.1021/ef2009265

[16] Renner TA (1988) Measurement and correlation of Diffusion coefficients for $\mathrm{CO}_{2}$ and rich-gas applications. SPE Reservoir Engineering 3: $517-$ 523. doi: 10.2118/15391-PA 
[17] Riazi MR, Whitson CH (1993) Estimating Diffusion coefficients of dense fluids. Industrial \& Engineering Chemistry Research 32: 30813088. doi: 10.1021/ie00024a018

[18] Zhang YP, Hyndmann CL, Maini BB (2000) Measurement of gas diffusivity in heavy oils. Journal of Petroleum Science and Engineering 25: 37-47. doi: 10.1016/S0920-4105(99)000315

[19] Sheikha H, Pooladi-Darvish M, Mehrotra AK (2005) Development of graphical methods for estimating the Diffusivity coefficient of gases in bitumen from pressure-decay data. Energy Fuels 19: 2041-2049. doi: 10.1021/ef050057c

[20] Rasmussen ML, Civan F (2009) Parameters of gas dissolution in liquids obtained by isothermal pressure decay. AIChE Journal 55: 9-23. doi: 10.1002/aic.11669

[21] Zheng S, Li HA, Sun H, Yang D (2016) Determination of Diffusion coefficient for alkane solvent- $\mathrm{Co}_{2}$ mixtures in heavy oil with consideration of swelling effect. Industrial \& Engineering Chemistry Research 55:15331549. doi: 10.1021/acs.iecr.5b03929

[22] Yang Z, Bryant S, Dong M, Hassanzadeh (2019) An analytical method of estimation Diffusion coefficients of gases in liquids from pressure decay tests. AIChE Journal 65: 434-445 doi: 10.1002/aic. 16408

[23] Rambaks A, Schmitz K (2019) Method for the experimental determination of the Bunsen absorption coefficient of hydraulic fluids. Paper presented at the symposium on fluid power and motion control, Sarasota, 7-9 October 2019

[24] Kratschun F, Schmitz K, Murrenhoff H (2016) Experimental investigation of the Bunsen and the Diffusion coefficients in hydraulic fluids. $10^{\text {th }}$ International Fluid Power Conference 181192

[25] Freudigmann H-A, Iben U, Pelz PF (2015) Air release measurements of $\mathrm{V}$-oil 1404 downstream of a micro orifice at choked flow conditions. Journal of Physics Conference Series 656. doi: 10.1088/1742-6596/656/1/012113

[26] Bird RB, Stewart WE, Lightfoot EN (2007) Transport Phenomena. New York, USA

[27] Whitaker S (2009) Derivation and application of the Stefan-Maxwell equations. Revista Mexicana de Ingeniería Química 8: 213 - 244.

[28] Vasserman AA, Kazavchinskii YaZ, Rabinovich VA (1966) Thermophysical properties of air and air components. Moscow, Soviet Union
[29] Wagner W, Kruse A (1998) Properties of Water and Steam. Berlin, Germany 